\title{
Macroscale Thermal Diode-Like Black Box with High Transient Rectification Ratio
}

\author{
Shiyao Huang, ${ }^{1}$ Jiawei Zhang, ${ }^{2}$ Meng Wang, ${ }^{3}$ Wei Lan, ${ }^{1}$ Run $\mathrm{Hu}^{1 *}$ and Xiaobing Luo ${ }^{1}$
}

Based on the transformation thermotics theory, many novel thermal functionalities have been achieved, such as thermal cloaking, concentrating, camouflaging, etc. Here, we propose a kind of macroscale thermal diode-like black box based on two typical outcomes of transformation thermotics - the energy shielding and harvesting units. The proposed macroscale thermal black box, acting as a thermal diode, creates a new record of transient thermal rectification ratio that can be as high as about 50, far breaking the highest record of 2.6 in the literature. Most existing thermal diodes are in the steady state realm, which, however, is not a necessary requirement. The enlightenment here lies in the recapture of the transient behaviors of thermal diode, which is more practical and promising in the thermal computation applications as it is time-consuming to wait for thermal equilibrium. The proposed macroscale thermal diode-like black box is believed to promote the implementation of thermal rectifier related applications, like thermal diode, thermal-logic operation, and phononics.

Keywords: Transformation thermotics; Thermal metamaterials; Thermal diode

Received 21 September 2019, Accepted 16 October 2019

DOI: $10.30919 /$ esee 8 c330

\section{Introduction}

Transformation thermotics theory, inspired from the seminal transformation optics theory, empowers us to design and fabricate thermal metamaterials for heat flow manipulation. ${ }^{1,2}$ The key feature of these thermal metamaterials lies in the anisotropic and inhomogeneous material makeup, thus they are not seen in nature. Thanks for the anisotropic feature, heat flow can be manipulated to realize directional conduction rather than omnidirectional diffusion, enabling many counterintuitive thermal phenomena and functionalities, such as thermal cloaking, ${ }^{3-14}$ concentrating, ${ }^{15-17}$ reflecting, ${ }^{18}$ refracting, ${ }^{19}$ camouflaging, ${ }^{20-24}$ lensing, ${ }^{25}$ encoding, ${ }^{26}$ etc. For a thermal cloak, the transformed thermal conductivity tensor, according to the transformation thermotics theory, changes to $\boldsymbol{\kappa}=\kappa_{0} \operatorname{diag}\left(\kappa_{r}, \kappa_{\theta}, 1\right)=\kappa_{0} \operatorname{diag}\left(\frac{r-a}{r}, \frac{r}{r-a}, 1\right)$ in the cylindrical coordinate system, where $\kappa_{0}$ is the thermal conductivity of the background plate, $r$ is the radius in the range of $[\mathrm{a}, \mathrm{b}]$, and $a$ and $b$ are the internal and exterior radii of the annular cloak structure. When $r$ approaches $a$, the radial component $\kappa_{\mathrm{r}}$ vanishes while the tangential component $\kappa_{\theta}$ tends to be infinite; as a result, the heat flow can be tuned to pass around central region without entering the central region, and then return back to the original direction, which seems as if heat conducts in a homogenous plate. Since no heat enters the central region, the local temperature is

\footnotetext{
${ }^{\prime}$ State Key Laboratory for Coal Combustion, School of Energy and Power Engineering, Huazhong University of Science and Technology, Wuhan 430074, China

${ }^{2}$ Well-Tech Research Institute of China Oilfield Service Limited, Beijing, 101149, China

${ }^{3}$ China-EU Institute for Clean and Renewable Energy, Huazhong University of Science and Technology (HUST), Wuhan, 430074, China

*E-mail: hurun@hust.edu.cn
}

expected to be maintained the same, free from the influence of the external temperature change. In such circumstance, any objects sitting in the central region, will be concealed from the external observers through an infrared (IR) camera. Such functionality draws most attentions and interests, especially for the optical counterpart, because it provides us a possibility to realize the invisibility cloaks that have only stayed in science fiction and movies for a long time. ${ }^{27-31}$ As for practical fabrication, the alternating concentric rings of homogeneous materials with distinct thermal conductivities are proposed and validated to be effective in thermal shielding from central region. Such practical structure is called an energy shielding unit (ESU) hereinafter. On the contrary, when changing $\boldsymbol{\kappa}$ from $\boldsymbol{\kappa}=\kappa_{0} \operatorname{diag}\left(\kappa_{r}, \kappa_{\theta}, 1\right)$ to $\boldsymbol{\kappa}=\kappa_{0} \operatorname{diag}\left(\kappa_{\theta}, \kappa_{r}, 1\right)$, we can realize the thermal concentrating effect, where heat tends to enter the central region because the radial component tends to infinite and the tangential component vanishes. To fabricate the thermal concentrator, the alternating sensu-shaped structures of two distinct materials are proposed to achieve thermal energy harvesting, thus it is called an energy harvesting unit (EHU) hereinafter. Based on the contrary local heat flux values inside the central regions in ESU and EHU, we proposed the thermal encoding concept to interpret as digits of 0 and 1 for thermal memory application. ${ }^{26}$ But beyond these heat distribution functionalities, what else can these typical outcomes of transformation thermotics, ESU and EHU, be used for?

In this study, we, based on ESU and EHU, turn to explore the possibility of thermal rectifier/diode application, which, analogous to asymmetric current characteristics in the electrical diode and lightemitting diode, can transport thermal energy asymmetrically and is expected to play an important role in the future thermal-logic operation and computation. ${ }^{32}$ Our modern world is greatly promoted by the rapid development of electronic-based computers, which, nevertheless, is hindered by the heat dissipation capability in the post-Moore nowadays. In contrast, the thermal computation can break such limit, thus thermal 
diode draws lots of attentions recently. To achieve a thermal diode, people have exploited the nonlinear phonon dynamics in anharmonic lattice structures, asymmetric nanostructures, ${ }^{33}$ near-/far-field thermal radiation regulation, ${ }^{34-37}$ phase-changing materials. ${ }^{37-40}$ Based on the transformation thermotics, $\mathrm{Li}$ et al. proposed a macroscopic thermal diode by treating materials with temperature-dependent conductivity. ${ }^{3}$ Their theory and simulations are beyond criticism, but their experimental validation is actually another story by resorting to the shape-memory alloy. As for thermal diode, the figure of merit is the thermal rectification ratio ${ }^{41}$

$$
\gamma=\frac{\Phi_{\mathrm{f}}}{\Phi_{\mathrm{r}}} \text { or } \gamma=\frac{\Phi_{\mathrm{f}}-\Phi_{\mathrm{r}}}{\Phi_{\mathrm{r}}}
$$

where $\Phi_{\mathrm{f}}$ and $\Phi_{\mathrm{r}}$ represent the heat-transfer rate in the forward- and reverse-temperature-biased scenarios, respectively. For consistent, we denote the thermal rectification ratio by the first definition and find that the majority $\gamma$ of the reported thermal diodes are as low as less than 2, which lays obstacles to make the thermal diode practical. Very recently, Cottrill et al. measured a dual phase change thermal diode with a thermal rectification ratio of 2.6, which was claimed to be the highest $\gamma$ in the literature. ${ }^{38}$ Such limited thermal rectification ratio sets up obstacles for practical applications. To tackle this issue, we design and fabricate a macroscale thermal black box, acting as a thermal diode, to achieve high- $\gamma$ by drawing inspiration from ESU and EHU. The performance, i.e. the thermal rectification ratio, is verified by both finiteelement simulations and experiments in transient and steady states, respectively. Proper physical explanations are discussed by varying the power of heat source, convection boundary conditions, with providing some suggestions on further improvement of the thermal rectification performance.

\section{Thermal diode-like black box structure}

The schematic of the proposed macroscale thermal Diode-like Black
Box is shown in Fig. 1. The key component is the asymmetric structure, which is a half ESU and a half EHU, which, as shown in Fig. 1d, is called as HSHH hereinafter. In the ESU half, concentric rings of copper and air layers are fabricated; while in the EHU half, sensu-shaped copper and air layers are manufactured. For such asymmetric HSHH, the thermal resistance from the central cylinder to the boundaries are distinct. To make up a thermal diode, we stack an EHU on the HSHH with very good contact in the central cylinders only. Four electrical heaters are mounted on the two sides of the EHU and the HSHH, respectively. With connecting with four electrical diodes, the forward current only heats up the heater on the left side of the top EHU, and the backward current only heats up the heater on the right side of the top EHU. The top EHU acts as the entrance for heat, while the down HSHH plays the role of exit. Two thermocouples are used to record the temperature of the exits. When heat enters the top EHU from the left entrance, as shown by the red dash in Fig. 1a, it will be conducted through the central contact and most will be conducted to the right exit. Thus the majority of heat can transport from the left to the right, which is denoted as the "FORWARD" state. On the contrary, when heat enters the top EHU from the right entrance, as shown by the red dash in Fig. $1 \mathrm{~b}$, the majority of heat will be conducted to the right side of HSHH unit and very little heat will transport to the left exit, which is denoted as the "REVERSE" state. Of course, heat will be conducted to the other side of the top EHU entrance, but it makes no difference to the "FORWARD/REVERSE" states. At the "FORWARD" state, heat (the majority) will transport from the left to the right; while at the "REVERSE" state, heat (the majority) will not transport from the right to the left. Such asymmetric characteristics of heat conduction shows the thermal rectification. From the working principle, we notice that the proposed macroscale thermal diode acts like a black box, in which the forward and backward current signals are transformed into the thermal signals for further thermal logic operation. Although it is not exactly the
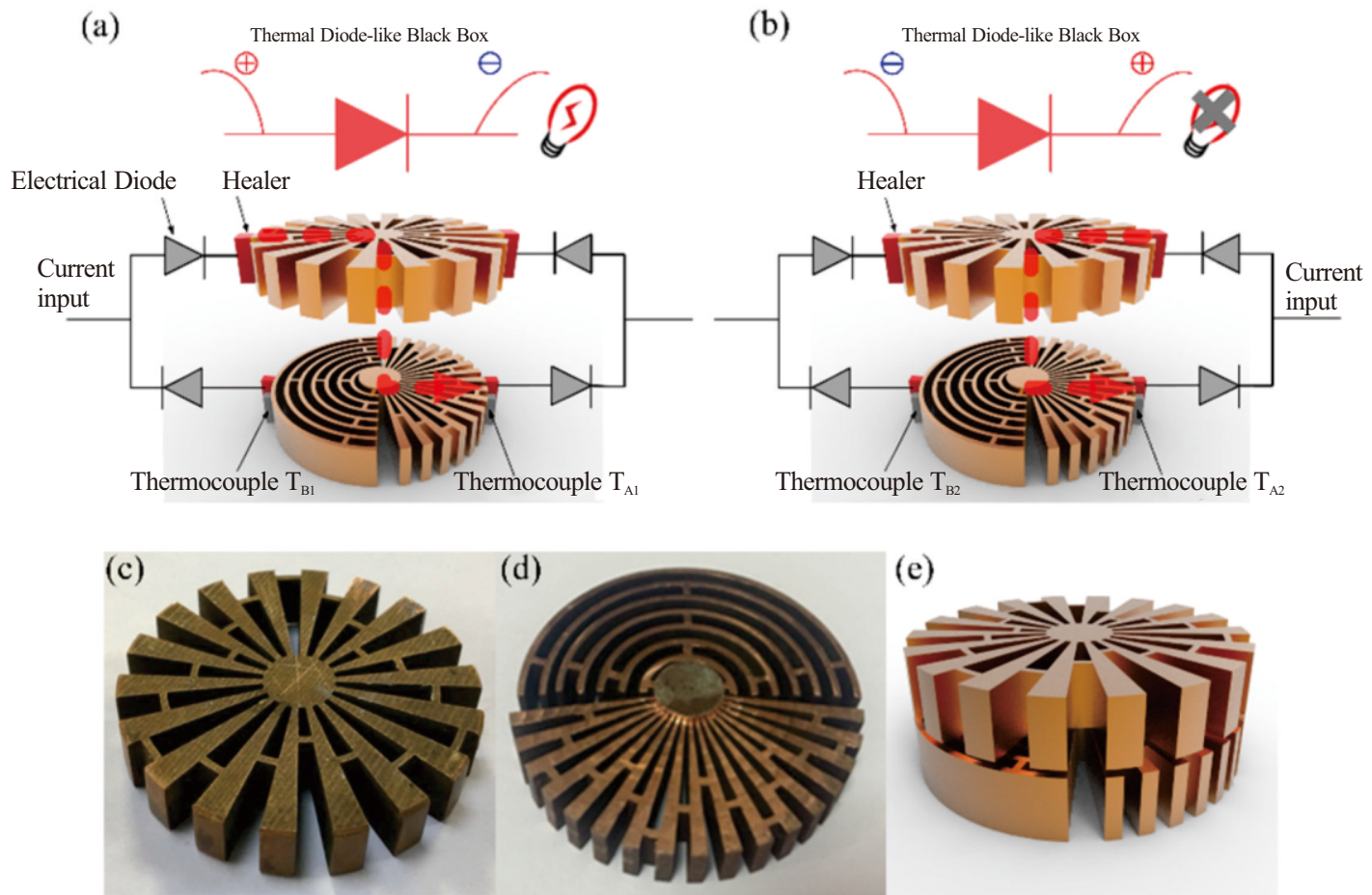

(e)

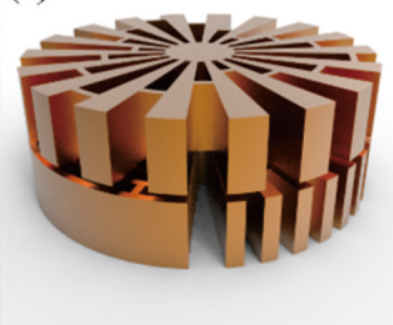

Fig. 1 Schematic for the working principle of the proposed thermal Diode-like Black Box. (a) Forward biased state: heat enters the top EHU from the left entrance, and conducts to the right exit of HSHH, which is denoted as the FORWARD state. (b) Reversed biased state: heat enters the top EHU from the right entrance, but very little heat conducts to the left exit of HSHH, which is denoted as the REVERSE state. (c-d) Images of the EHU and the HSHH. (e) Image of the thermal Diode-like Black Box. 
common sense of a diode, it still works well, as a whole, in the demonstrations of the "FORWARD" and "REVERSE" states of thermal rectification.

\section{Results and discussions}

To validate the thermal rectification performance of the proposed macroscale thermal Diode-like Black Box, we fabricate experimental samples by computer-numerical-control machine. The images of the EHU and HSHH are shown in Fig. 1c and 1d, respectively. The whole dimensions of these samples are consistent as $25 \mathrm{~mm}$ in radius and $9 \mathrm{~mm}$ in thickness. The thickness of the central cylinder of the HSHH is $1 \mathrm{~mm}$ thicker to avoid the complete contact between EHU and HSHH. After fabricating the EHU and HSHH separately, a thin layer of thermal paste is used to attach these two structures to reduce the contact thermal resistance.
For the experimental setup, two ceramic heating elements are attached to the entrance on the top EHU as the heat source. Four thermocouples are used to detect the temperature of the two sides of the HSHH, i.e. $T_{\mathrm{A}}$ and $T_{\mathrm{B}}$ in Fig. 1a and $1 \mathrm{~b}$. The input current is $1 \mathrm{~A}$ and input voltage is $3.7 \mathrm{~V}$, thus the power of heat source is 3.7 Watt. The measured temperature $\left(T_{\mathrm{A}}\right.$ and $T_{\mathrm{B}}$ ) changes are shown in Fig. 2a. It is seen that with time elapses, $T_{\mathrm{A}}$ and $T_{\mathrm{B}}$ increase quickly, followed by a plateau after 4,000 seconds. The key point in Fig. $2 \mathrm{a}$ is that $T_{\mathrm{A}}$ is always larger than $T_{\mathrm{B}}$, and the temperature difference in the end is about $12.5^{\circ} \mathrm{C}$. To better understand and interpret the experiments, we also do the finite-element simulations. The threedimensional model is established to be the same structures exactly as those in the experiments. The power of the heat source is also set as 3.7 Watts, the natural convection coefficient is $2 \mathrm{~W} /\left(\mathrm{m}^{2} \mathrm{~K}\right)$, and the origin temperature of the model is also $28^{\circ} \mathrm{C}$. The simulated $T_{\mathrm{A}}$ and $T_{\mathrm{B}}$ are also

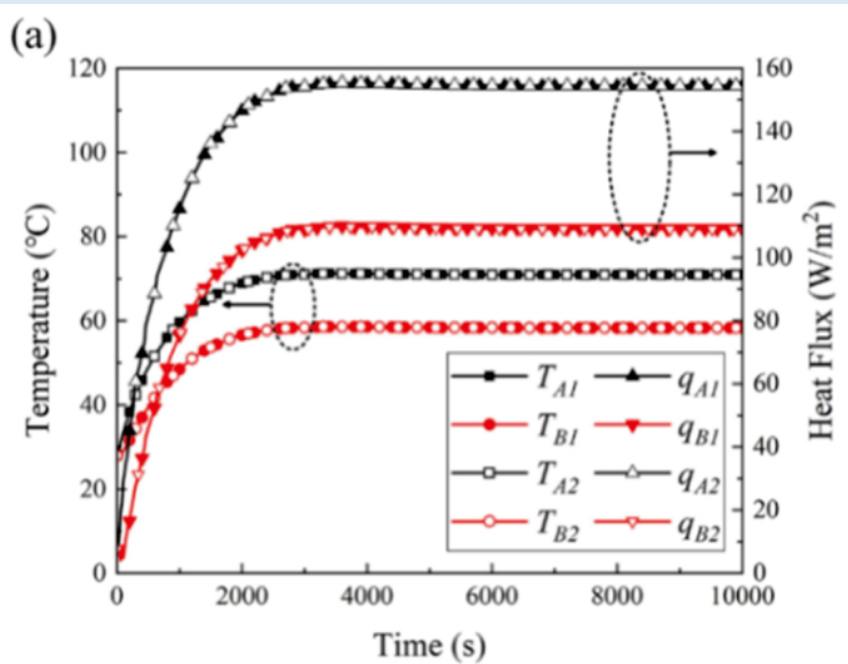

(b)

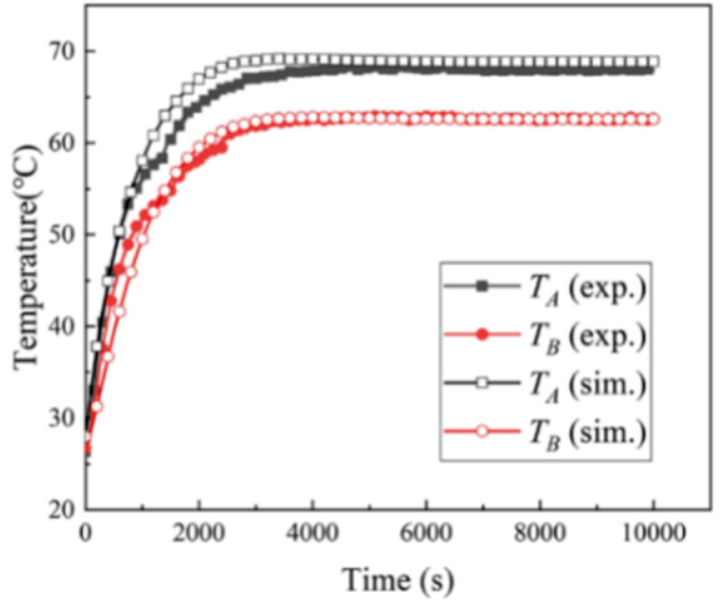

Fig. 2 (a) Temperature and heat flux curves of the two sides $\left(T_{\mathrm{A}}\right.$ and $\left.T_{\mathrm{B}}\right)$ of the HSHH units at the FORWARD and REVERSE states, and (b) temperature comparison of $T_{\mathrm{A}}$ and $T_{\mathrm{B}}$ between simulations and experiments.
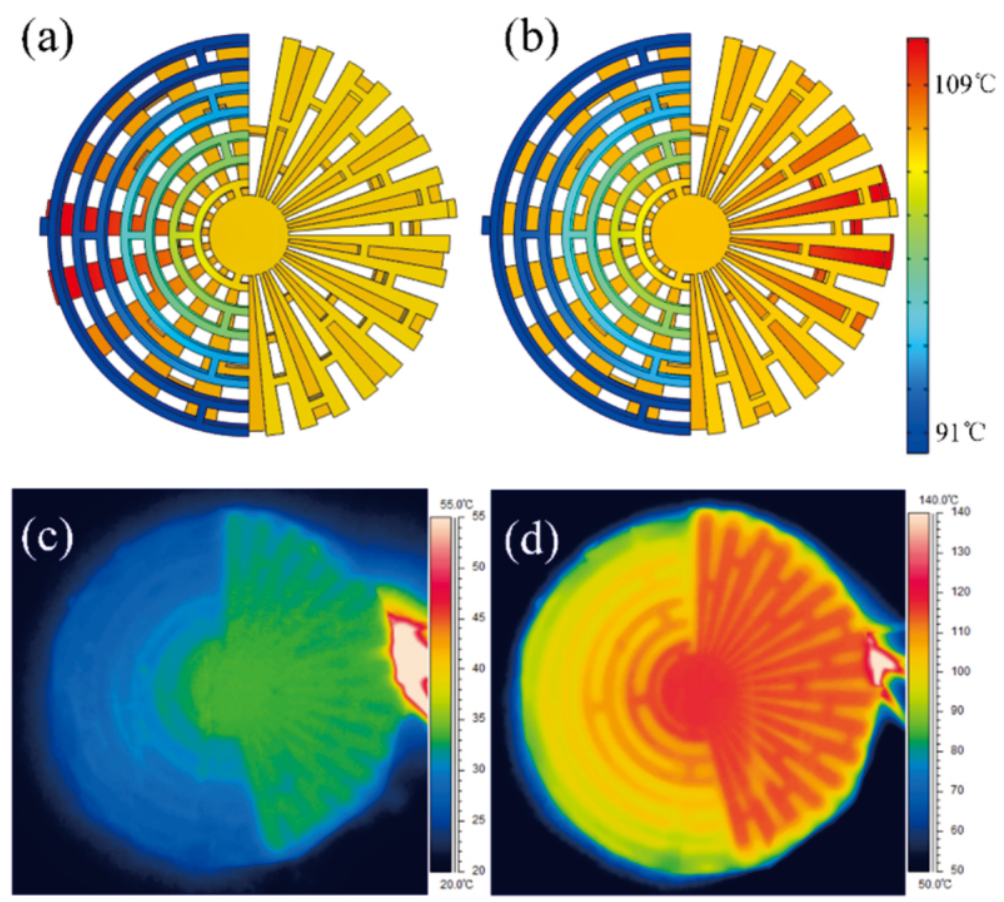

Fig. 3 Bottom view of the simulated temperature profiles of the macroscale thermal Diode-like Black Box at (a) FORWARD and (b) REVERSE working steady states. Bottom view of the experimental temperature profiles at REVERSE working states of the macroscale thermal Diode-like Black Box at (c) $t=1$ minute and (d) steady state. 
plotted in Fig. 2a, which agree with the experimental temperature curves very well. We also simulated the $T_{\mathrm{A}}$ and $T_{\mathrm{B}}$ at the "FORWARD" and "REVERSE" states, respectively. There is no accident that the $T_{\mathrm{Al}}$ and $T_{\mathrm{A} 2}, T_{\mathrm{B} 1}$ and $T_{\mathrm{B} 2}$ overlap with each other, because the thermal resistances of the system are fixed, and the only difference is the entrance location of input heat. To calculate the thermal rectification ratio, $\gamma$, we extract the local normal heat flux at the exits, denoted as $q_{\mathrm{A}}$ and $q_{\mathrm{B}}$, which are also plotted in Fig. 2b. It is seen that $q_{\mathrm{A} 1}$ and $q_{\mathrm{A} 2}, q_{\mathrm{B} 1}$ and $q_{\mathrm{B} 2}$ overlap with each other well and $q_{\mathrm{A}}$ is much larger than $q_{\mathrm{B}}$. In the end, the heat flux difference is as high as about $50 \mathrm{~W} / \mathrm{m}^{2}$. Both the temperature curves and the heat flux curves validate that the majority of heat will transport to the right exit of the HSHH, implying the asymmetric thermal rectification of the macroscale thermal Diode-like Black Box. The top views of the simulated temperature fields of thermal Diode-like Black Box at "FORWARD" and "REVERSE" states are shown in Fig. 3. It is seen clearly that no matter the heat enters from the left or the right entrance, the majority of heat goes to the right side of bottom HSHH, leaving the left side at relatively low temperature. The left-to-right "FORWARD" state and the right-to-left "REVERSE" state are the key feature of the macroscale thermal Diode-like Black Box. The bottom views of the experimental temperature profiles in Fig. $3 \mathrm{c}$ and $3 \mathrm{~d}$, show that even at transient state, the majority of heat flows to the EHU sides, which, benefit to the transient thermal rectification ratio, will be discussed later.
At steady state, the thermal rectification ratio $\gamma$, according to the definition, can be calculated as

$$
\gamma=\frac{\Phi_{\mathrm{f}}}{\Phi_{\mathrm{r}}}=\frac{\Phi_{\mathrm{A}}}{\Phi_{\mathrm{B}}}=\frac{h A_{\text {exit }}\left(T_{A}-T_{\infty}\right)}{h A_{\text {exit }}\left(T_{B}-T_{\infty}\right)}=\frac{h A_{\text {exit }} \theta_{\mathrm{A}}}{h A_{\text {exit }} \theta_{\mathrm{B}}}=\frac{q_{\mathrm{A}}}{q_{\mathrm{B}}}=\frac{\theta_{\mathrm{A}}}{\theta_{\mathrm{B}}}
$$

where $h$ is the convection coefficient, $A_{\text {exit }}$ is the exit area, $T_{\infty}$ is the ambient air temperature, and $\theta$ is the excess temperature accordingly. The excess temperature variations at different heat source powers and convective coefficients at steady and transient states are plotted in Figs. $4 \mathrm{a}$ and $4 \mathrm{~b}$, respectively. It is seen that with the increase of heat source power or the convective coefficient, the excess temperatures, $\theta_{\mathrm{A}}$ and $\theta_{\mathrm{B}}$ increase monotonously. When the convective coefficient is $2 \mathrm{~W} /\left(\mathrm{m}^{2} \mathrm{~K}\right)$, the transient $\theta_{\mathrm{A}}$ and $\theta_{\mathrm{B}}$ are shown in Fig. $4 \mathrm{~b}$, which indicates that with time going by, the excess temperature increases firstly and then achieves stable values with different equilibrium time at different heat source powers. The thermal rectification ratio $\gamma$ is calculated according to Eq. (1) and plotted in Fig. $4 \mathrm{c}$ and $4 \mathrm{~d}$ for the steady and transient states. For the steady state, we also calculate the ratio $\gamma$ by Eq. (2), which are exactly the same as the results by Eq. (1), thus we don't repeat in Fig. 4c. It is seen in Fig. 4c that $\gamma$ increases with the convective coefficient, but is independent on the heat source power. The reason can be explained by the heat loss analysis. As shown in Fig. 5a, the input heat is dissipated by following parts: heat loss to the air $\left(Q_{\mathrm{h}}\right.$, not shown), heat accumulation in the four parts of the macroscale thermal Diode-like Black Box $\left(Q_{1}, Q_{2}, Q_{3}\right.$, and $\left.Q_{4}\right)$. Within a (a)

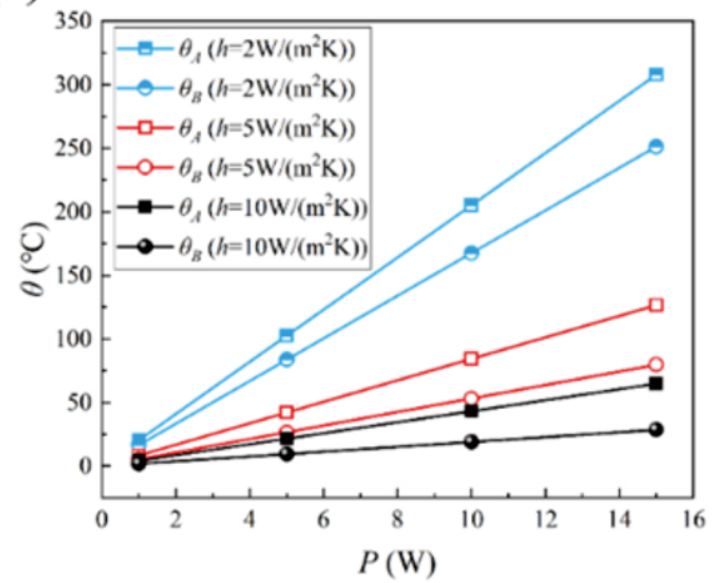

(c)

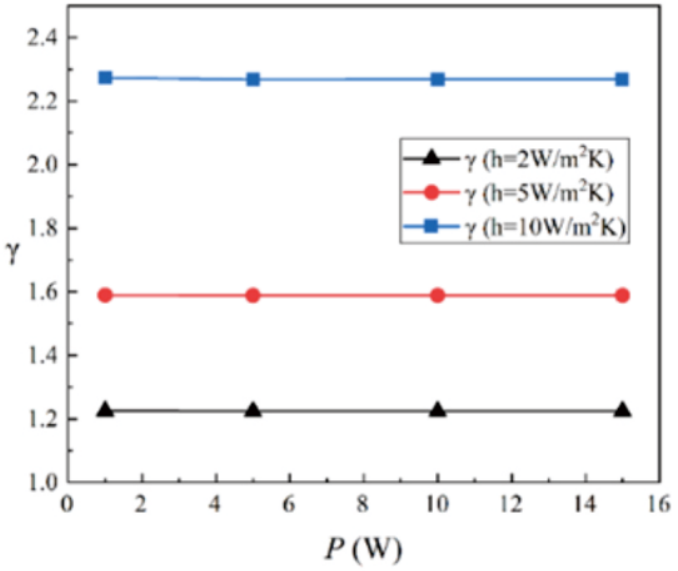

(b)

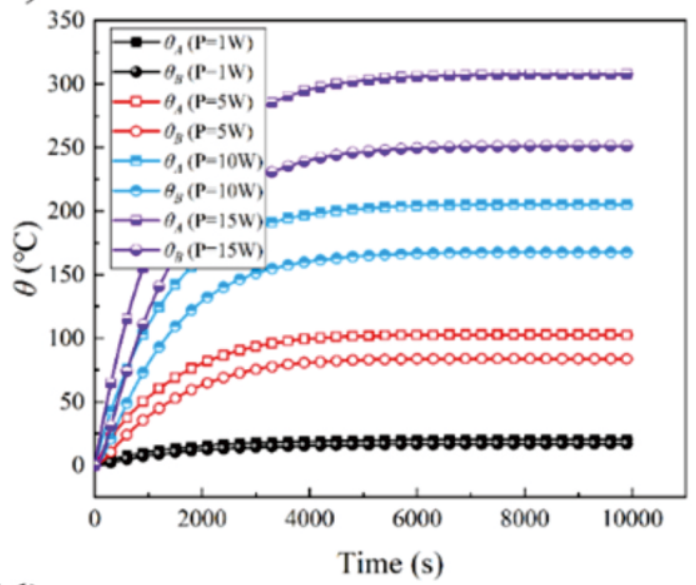

(d)

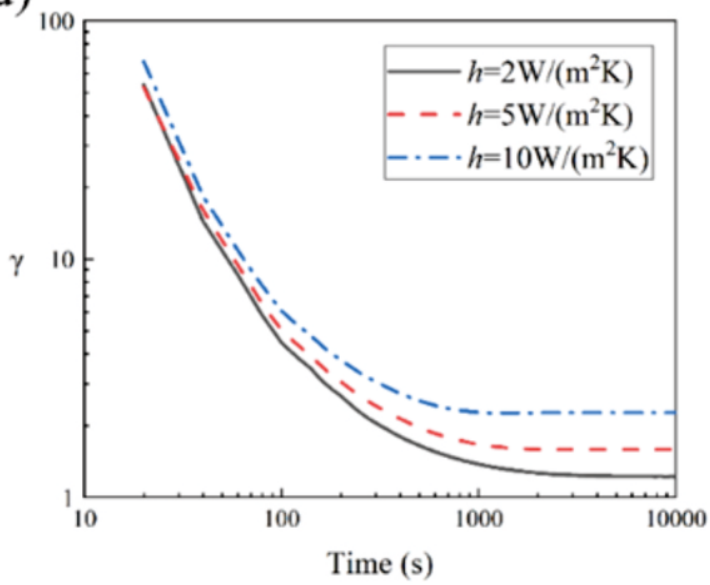

Fig. 4 Excess temperature of $T_{\mathrm{A}}$ and $T_{\mathrm{B}}$ variations on different heat source powers and convective coefficients at (a) steady and (b) transient states. (c) The thermal rectification ratio $\gamma$ on different heat source powers and convective coefficients at steady states. (d) The thermal rectification ratio $\gamma$ on different heat source powers and convective coefficients at transient states. 
given time $\tau, Q_{\mathrm{h}}$ can be calculated as $Q_{\mathrm{h}}=\int_{0}^{\tau} \int_{A} h \theta_{\tau} d A d \tau$, where $A$ is the total surface area of the macroscale diode-like black box, $\theta_{\tau}$ is the excess temperature under various time. The heat accumulations in the macroscale thermal diode-like black box are calculated as $Q_{i}=\int_{t}^{t} \int_{V} \rho_{i} c_{i} d V d t$, where $\rho, c$ and $V$ are the density, specific heat, and volume of each part, and the subscript $i$ denotes the each part in Fig. 5a. For the given time 6,000 seconds, the integrated heat loss at constant input power $P=10$ $\mathrm{W}$, or at the constant convective coefficient $h=2 \mathrm{~W} /\left(\mathrm{m}^{2} \mathrm{~K}\right)$ are illustrated as pie charts in Fig. 5b, respectively. At constant $P=10 \mathrm{~W}$, it is seen that with the increase of $h$, the heat loss $Q_{\mathrm{h}}$ increases from $79 \%$ to $95 \%$ monotonously, which can be understood by resorting to the Newton's law. While at constant $\mathrm{h}, Q_{\mathrm{h}}$ is almost constant and independent on $P$, which is because that the increase of $P$ leads to the increase of all heat loss with subequal magnitude, and the percentage of $Q_{\mathrm{h}}$ is almost the same. We also plot the ratio of $Q_{4} / Q_{3}$ versus $h$ and $P$ in Fig. 5c. It is seen that the ratio of $Q_{4} / Q_{3}$ versus increases relatively significantly with the increase of $h$ but is almost independent on $P$. Although ratio of $Q_{4} / Q_{3}$ is not the thermal rectification ratio $\gamma$ of the thermal diode-like black box, the trend should be similar since the exits are located on the two boundaries of $Q_{4}$ and $Q_{3}$. According to Eq. (1), the thermal rectification ratio $\gamma$ is only dependent on the excess temperature, which has been illustrated in Fig. 4a. Extracting the excess temperature from Fig. 4a can be used to explain Fig. 4c quantitatively, and Fig. 5 can be used to explain Fig. 4c qualitatively.

Compared to the steady state thermal rectification ratio, the transient one is more interesting. It is seen in Fig. $4 d$ that with time going by, the rectification ratio decreases sharply from several tens and achieves to its steady value after 1,000 seconds. The increase of convective coefficient $h$ leads to the enhancement of rectification ratio, which is consistent with that in Fig. 4c. Note that most existing thermal rectification ratios are less than 3, the transient one here can exceeds 10 , or even can be as high as 50. Actually, we don't need to wait for the steady state in the real application, especially for the thermal computing, thus the transient thermal rectification is enough to help for the thermal-logic operation. For practical applications, we can design some thermal-logic circuit units to extract the thermal signals (ON/OFF) based on the temperature or heat flux information. The superhigh thermal rectification has never been reported, thus we believe it will benefit for the promotion of feasible macroscale thermal diode-like black box. To further enhance the rectification ratio, a possible strategy is to design the $\mathrm{HSHH}$ with two different materials. For instance, if the half-ESU is made of material with lower thermal conductivity, it is perceived that the thermal rectification ratio can be increased further at both steady and transient states.

\section{Conclusions}

In conclusion, we propose a macroscale thermal diode-like black box, via energy shielding and harvesting units, to achieve high transient thermal rectification ratio. The macroscale diode-like black box, consisting of the energy shielding and harvesting units, acts as a thermal diode, where the forward and backward current input generate the thermal rectification. Both steady and transient simulations were conducted to analyze the thermal rectification process, with comparison with the experimental results. Compared with the steady rectification ratio, we find the transient ratio can be as high as 50 , which is a new record compared with the existing ones in the literature. The transient one is more important and useful, avoiding the waiting for thermal equilibrium, which will benefit for the thermal computing and thermallogic operation.

\section{Acknowledgements}

The authors would like to acknowledge the financial support by National Natural Science Foundation of China (51606074, 51625601), and the Ministry of Science and Technology of the People's Republic of China (2017YFE0100600).

\section{References}

1. J. B. Pendry, D. Schurig and D. R. Smith, Science, 2006, 312, 1780-1782.

2. C. Z. Fan, Y. Gao and J. P. Huang, Appl. Phys. Lett., 2008, 92, 251907.

3. Y. Li, X. Shen, Z. Wu, J. Huang, Y. Chen, Y. Ni and J. Huang, Phys. Rev. Lett., 2015, 115, 195503.

4. D. M. Nguyen, H. Xu, Y. Zhang and B. Zhang, Appl. Phys. Lett., 2015, 107, 121901.

5. H. Xu, X. Shi, F. Gao, H. Sun and B. Zhang, Phys. Rev. Lett., 2014, 112 054301 .
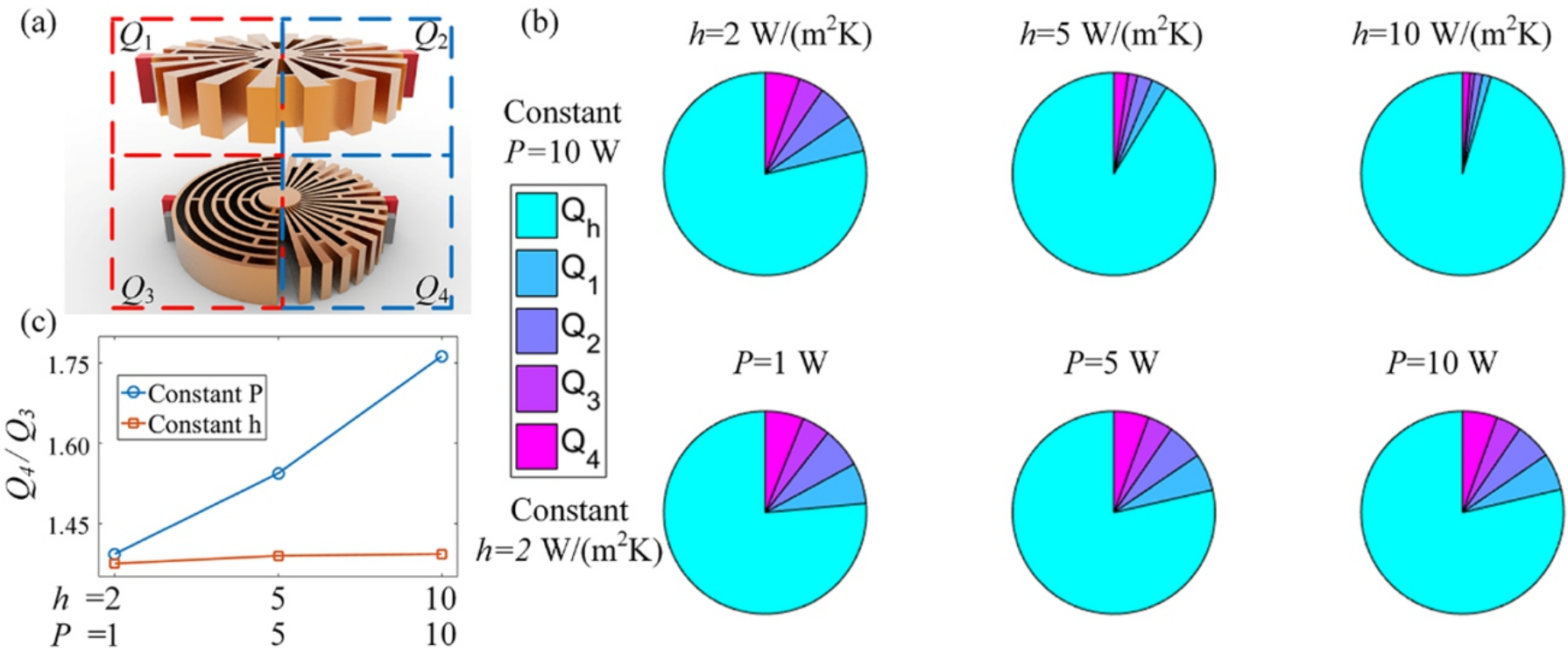

Fig. 5 (a) Schematic for heat loss analysis. (b) Heat loss percentage at different convective coefficients (first row), and at different input powers (second row). (c) Dependence of $Q_{4} / Q_{3}$ on convective coefficient $h$ and input power $P$. 
6. L. Wu, J. Heat Transfer, 2015, 137, 021301.

7. T. Han, X. Bai, D. Gao, J. T. L. Thong, B. Li and C. W. Qiu, Phys. Rev. Lett., 2014, 112, 054302.

8. Y. Zhang, H. Xu and B. Zhang, AIP Adv., 2015, 5, 053402.

9. A. Alù, Phys., 2014, 7, 12.

10. Y. Liu, Y. Cheng, R. Hu and X. Luo, Phys. Lett. A, 2019, 383, 2296-2301.

11. R. Hu and X. Luo, Natl. Sci. Rev., 2019, nwz114, DOI: 10.1093/nsr/nwz114

12. T. Han, P. Yang, Y. Li, D. Lei, B. Li, K. Hippalgaonkar and C. W. Qiu, Adv. Mater., 2018, 30, 1804019.

13. L. Zhou, S. Huang, M. Wang, R. Hu and X. Luo, Phys. Lett. A, 2019, 383, 759-763.

14. R. Wu, T. Hong, Q. Cheng, H. Zou, Y. Fan and X. Luo, Int. J. Heat Mass Tran., 2019, 137, 42-51.

15. F. Chen and D. Y. Lei, Sci. Rep., 2015, 5, 11552.

16. S. Narayana and Y. Sato, Phys. Rev. Lett., 2012, 108, 214303.

17. C. Lan, B. Li and J. Zhou, Opt. Express, 2015, 23, 3478.

18. R. Hu, S. Zhou, W. Shu, B. Xie, Y. Ma and X. Luo, AIP Adv., 2016, 6, 125111.

19. R. Hu, B. Xie, J. Hu, Q. Chen and X. Luo, EPL, 2015, 111, 54003.

20. T. Han, X. Bai, J. T. L. Thong, B. Li and C. W. Qiu, Adv. Mater, 2014, 26, 1731.

21. R. Hu, S. Zhou, Y. Li, D. Y. Lei, X. Luo and C. W. Qiu, Adv. Mater, 2018, 30, 1707237 .

22. O. Salihoglu, H. B. Uzlu, O. Yakar, S. Aas, O. Balci, N. Kakenov, S. Balci, S. Olcum, S. Süzer and C. Kocabas, Nano Lett., 2018, 18, 4541-4548.

23. J. Qin, W. Luo, P. Yang, B. Wang, T. Deng and T. Han, Int. J. Heat Mass Transf., 2019, 141, 487-490.

24. S. Zhou, R. Hu and X. Luo, Int. J. Heat Mass Transf., 2018, 127, 607-613.

25. Y. Liu, F. Sun and S. He, Opt. Express, 2016, 24, 5683-5692.

26. R. Hu, S. Huang, M. Wang, L. Zhou, X. Peng and X. Luo, Phys. Rev. Appl.,
2018, 10, 054032 .

27. R. Schittny, M. Kadic, T. Bueckmann and M. Wegener, Science, 2014, 345, 427-429.

28. U. Leonhardt. To invisibility and beyond, Nature, 2011, 471, 292.

29. S. Zhang, C. Xia and N. Fang, Phys. Rev. Lett., 2011, 106, 024301.

30. R. Hu, S. Zhou, X. Yu and X. B. Luo, J. Phys. D: Appl. Phys., 2016, 49, 415302.

31. F. Gomory, M. Solovyov, J. Souc, C. Navau, J. Prat-Camps, A. Sanchez, F. Goemoery, M. Solovyov, J. Souc, C. Navau, J. Prat-Camps and A. Sanchez, Science, 2012, 335, 1466.

32. N. Li, J. Ren, L. Wang, G. Zhang, P. Hänggi and B. Li, Rev. Mod. Phys., 2012, 84, 1045.

33. Y. Wang, A. Vallabhaneni, J. Hu, B. Qiu, Y. P. Chen and X. Ruan, Nano Lett., 2014, 14, 592

34. C. R. Otey, W. T. Lau and S. Fan, Phys. Rev. Lett., 2010, 104, 154301.

35. S. Jia, Y. Fu, Y. Su and Y. Ma, Opt. Lett., 2018, 43, 5619.

36. A. Fiorino, D. Thompson, L. Zhu, R. Mittapally, S. A. Biehs, O. Bezencenet, N. El-Bondry, S. Bansropun, P. Ben-Abdallah, E. Meyhofer and P. Reddy, ACS Nano, 2018, 12, 5174.

37. H. Kang, F. Yang and J. J. Urban, Phys. Rev. Appl., 2018, 10, 024034.

38. A. L. Cottrill, S. Wang, A. T. Liu, W. J. Wang and M. S. Strano, Adv. Energy Mater, 2018, 8, 1702692.

39. E. Pallecchi, Z. Chen, G. E. Fernandes, Y. Wan, J. H. Kim and J. Xu, Mater. Horizons, 2015, 2, 125.

40. A. L. Cottrill and M. S. Strano, Adv. Energy Mater, 2015, 5, 1500921.

41. H. Tian, D. Xie, Y. Yang, T. Ren, G. Zhang, Y. Wang, C. Zhou, P. Peng, L. Wang and L. Liu, Sci. Rep., 2012, 2, 523.

Publisher's Note Engineered Science Publisher remains neutral with regard to jurisdictional claims in published maps and institutional affiliations. 